02

\title{
Механизм зависимости флуоресцентных свойств тетраарилтетрацианопорфиразина и его производных от вязкости
}

\author{
(C) Н.В. Ивашин \\ Институт фризики им. Б.И. Степанова НАН Беларуси, \\ 220072 Минск, Республика Беларусь \\ e-mail: inv_vni@mail.ru
}

Поступила в редакцию 02.07.2021 г.

В окончательной редакции 19.08.2021 г.

Принята к публикации 31.08.2021 г.

\begin{abstract}
Проведены квантово-химические расчеты конформационной структуры в основном и в возбужденных состояниях тетраарилтетрацианопорфиразина $\left(\mathrm{H}_{2}-\mathrm{Pz}(\mathrm{Ph})_{4}(\mathrm{CN})_{4}\right)$ и его производных, которые рассматриваются в качестве перспективных фотосенсибилизаторов фотодинамического действия с функцией флуоресцентного контроля степени разрушения раковых клеток. Показано, что в отсутствие специфических взаимодействий с растворителем для данных соединений характерна плоская структура макроцикла как в основном, так и возбужденном $S_{1}$-состоянии. Среди низколежащих возбужденных состояний отсутствуют таковые, заселение которых может приводить к тушению флуоресценции за счет заметного изменения положения фенильных колец относительно макроцикла по торсионной координате. Это позволяет исключить отнесение данных соединений к флуоресцентным роторам, как это предполагалось ранее. Установлено, что $\mathrm{H}_{2}-\mathrm{Pz}(\mathrm{Ph})_{4}(\mathrm{CN})_{4}$ и его производные в растворе образуют сольватные комплексы с кислородсодержащими молекулами растворителя (вода, метанол, этанол, глицерин, тетрагидрофуран) с участием атомов Н и $\mathrm{N}$ пиррольных и пирролениновых колец соответственно. Для данных комплексов характерно неплоскостное искажение макроцикла, которое существенно увеличивается в $S_{1}$-состоянии и приводит к большим смещениям периферических заместителей перпендикулярно макроциклу. Конформационная динамика в $S_{1}$-состоянии сопровождается уменьшением энергетического зазора $\Delta E\left(S_{0}-S_{1}\right)$, увеличением спин-орбитального взаимо-

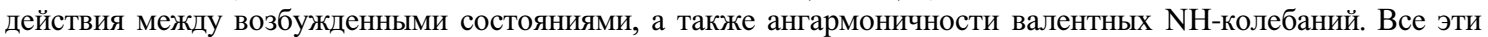
факторы приводят к уменьшению времени жизни флуоресценции $\left(\tau_{\mathrm{F}}\right) \mathrm{H}_{2}-\mathrm{Pz}(\mathrm{Ph})_{4}(\mathrm{CN})_{4}$ и их производных в растворе, способствуют зависимости $\tau_{\text {F }}$ от вязкости среды.
\end{abstract}

Ключевые слова: тетраарилтетрацианопорфиразин, производные тетраарилтетрацианопорфиразина, конформационная структура, квантово-химические расчеты.

DOI: $10.21883 /$ OS.2021.12.51739.2507-21

\section{Введение}

Возможность использования фотосенсибилизатора фотодинамической терапии (ФДТ) для контроля степени разрушения раковых клеток была продемонстрирована относительно недавно [1,2]. В качестве фотосенсибилизатора авторы использовали искусственный димер двух порфиринов, соединенных между собой бутиленовым мостиком через мезоположения макроциклов. Его флуоресцентные свойства в растворе, в частности положение максимума полосы флуоресценции, существенно зависят от вязкости среды. Наличие такой особенности у данного соединения позволило показать, что в процессе ФДТ и разрушения раковой клетки происходит заметное увеличение вязкости ее внутриклеточной среды. Для некоторых модификаций данного соединения была обнаружена также зависимость флуоресцентных свойств от температуры [3]. Проведенное недавно исследование показало хорошее соответствие результатов, полученных в модельных условиях и in vivo [4]. На основании этих работ были предложены методы [4], позволяющие осуществлять динамический мониторинг изменения вяз- кости в клетках раковой опухоли животных в процессе ФДТ с целью контроля степени ее разрушения.

Следует отметить, что использованный в работах $[1,2]$ искусственный порфириновый димер представляет собой довольно сложную и дорогую с точки зрения синтеза конструкцию. Более простые и дешевые фотосенсибилизаторы-сенсоры были предложены на основе тетраарилтетрацианопорфиразина $\left(\mathrm{H}_{2}-\mathrm{Pz}(\mathrm{Ph})_{4}(\mathrm{CN})_{4}\right)$ и его производных [5-8]. Эти соединения отличаются от порфиразина наличием заместителей $\mathrm{C}_{6} \mathrm{H}_{5}$ и $\mathrm{C} \equiv \mathrm{N}$ на пиррольных и пирролениновых кольцах (рис. 1). Для них была обнаружена зависимость времени жизни и квантового выхода флуоресценции от вязкости растворителя [6,7]. Было показано, что такие соединения сочетают в себе функции фотосенсибилизатора и флуоресцентного сенсора, хорошо накапливаются в околоядерной области опухолевых клеток и обладают фотодинамическим эффектом.

За последние несколько десятилетий было синтезировано множество фотосенсибилизаторов образования синглетного кислорода, из которых лишь некоторые оказались пригодными для внедрения в медицинскую 


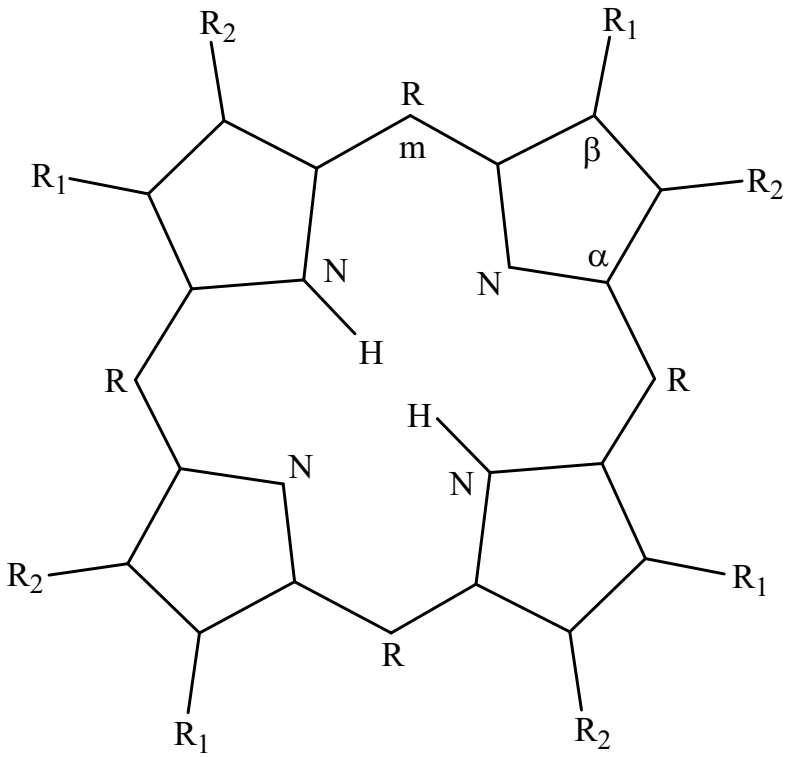

Рис. 1. Структурные формулы исследованных соединений. $\mathrm{H}_{2}-\mathrm{Pz}: \mathrm{R}=\mathrm{N}, \mathrm{R}_{1}=\mathrm{R}_{2}=\mathrm{H} ; \mathrm{H}_{2}-\mathrm{Pz}(\mathrm{Ph})_{4}(\mathrm{CN})_{4}: \mathrm{R}=\mathrm{N}, \mathrm{R}_{1}=\mathrm{Ph}$, $\mathrm{R}_{2}=\mathrm{CN} ; \mathrm{H}_{2}-\mathrm{Pz}(\mathrm{Ph})_{4}: \mathrm{R}=\mathrm{N}, \quad \mathrm{R}_{1}=\mathrm{Ph}, \mathrm{R}_{2}=\mathrm{H} ; \mathrm{H}_{2}-\mathrm{Pz}(\mathrm{CN})_{4}$ : $\mathrm{R}=\mathrm{N}, \mathrm{R}_{1}=\mathrm{H}, \mathrm{R}_{2}=\mathrm{CN} ; \mathrm{H}_{2}-\mathrm{Pz}(\mathrm{p}-\mathrm{F}-\mathrm{Ph})_{4}(\mathrm{CN})_{4}: \mathrm{R}=\mathrm{N}, \mathrm{R}_{1}=\mathrm{p}-$ $\mathrm{F}-\mathrm{Ph}, \mathrm{R}_{2}=\mathrm{CN} ; \mathrm{H}_{2}-\mathrm{Pz}\left(\mathrm{o}-\mathrm{CH}_{3}-\mathrm{Ph}\right)_{4}(\mathrm{CN})_{4}: \mathrm{R}=\mathrm{N}, \mathrm{R}_{1}=\mathrm{o}-\mathrm{CH} 3-\mathrm{Ph}$, $\mathrm{R}_{2}=\mathrm{CN} ; \mathrm{H}_{2}-\mathrm{P}(\mathrm{Ph})_{4}(\mathrm{CN})_{4}: \mathrm{R}=\mathrm{CH}, \mathrm{R}_{1}=\mathrm{Ph}, \mathrm{R}_{2}=\mathrm{CN}$. Приведены принятые для тетрапиррольного макроцикла обозначения атомов в мезо-положении $(m)$ и на пиррольных и пирролениновых кольцах $(\alpha, \beta)$.

практику. Хороший фотосенсибилизатор для ФДТ, как известно, должен соответствовать целому ряду фотофизических, спектроскопических, биологических и химикотехнологических критериев [9]. Среди них важнейшими являются следующие: высокий квантовый выход генерации синглетного кислорода, низкая темновая токсичность, высокая селективность накопления в опухолях, сильное поглощение в области прозрачности биологических тканей, быстрое выведение из здоровых тканей. Этим же критериям должны соответствовать и фотосенсибилизаторы-сенсоры. Разработка препаратов такого типа находится на начальной стадии и является актуальной проблемой. Решение связанных с этим задач требует глубокого понимания механизмов влияния среды на флуоресцентные свойства предложенных к настоящему времени и потенциальных фотосенсибилизаторовсенсоров. Установление таких механизмов будет способствовать также поиску путей структурной модификации уже известных фотосенсибилизаторов ФДТ с целью придания им свойств сенсоров.

Зависимость флуоресцентных свойств $\mathrm{H}_{2}-\mathrm{Pz}(\mathrm{Ph})_{4}(\mathrm{CN})_{4} \quad$ и $\quad$ его производных $\quad$ от $\quad$ вязкости ранее была связана с их отнесением к так называемым молекулярным роторам. При этом было предположено, что флуоресцентные свойства варьируют за счет изменения положения фенильных колец по торсионной координате и заселения нефлуоресцирующего состояния неизвестной природы.

Целью данной работы было установление механизма влияния вязкости на флуоресцентные свойства $\mathrm{H}_{2}$ $\mathrm{Pz}(\mathrm{Ph})_{4}(\mathrm{CN})_{4}$ и его модификаций на основе молекулярного моделирования их структуры в основном и в возбужденных состояниях с учетом макроскопических и специфических взаимодействий с растворителем.

\section{Методы}

Расчеты выполнялись с использованием квантовохимического пакета программ Gaussian 09 (D.01) [10]. Оптимизация геометрии, расчет электронных переходов и колебаний проводились преимущественно с функционалом wB97XD [11]. Правомочность его выбора ранее нами была продемострирована для ряда тетрапирролов при расчете электронных переходов с переносом заряда [12], геометрии сольватных комплексов в основном и в возбужденных состояниях [13]. Для сравнения структура сольватных комплексов $\mathrm{H}_{2}-\mathrm{Pz}(\mathrm{Ph})_{4}(\mathrm{CN})_{4}$ и его производных рассчитывалась также с функционалом B3LYPDCP [14]. В нем используются потенциалы гауссова типа, которые позволяют скорректировать распределение электронной плотности на больших расстояниях от атомных центров. Это позволяет улучшить результаты расчетов структуры различных ван-дер-ваальсовых комплексов и, в частности, образованных за счет $\pi-\pi$ взаимодействий. В соответствии с рекомендациями авторов [14] расчеты проводились с базисным набором 6-31+G(d,p) на всех атомах. Больших отличий в структуре сольватных комплексов, рассчитанных с функционалами wB97XD и B3LYP-DCP, обнаружено не было.

В расчетах вертикальных электронных переходов и структуры молекул в возбужденных состояниях применялся метод TDDFT. Учет влияния среды на структуру и спектроскопические свойства соединений и их комплексов проводился в рамках модели поляризуемого континуума (РСМ) [15].

Структура исследованных систем, молекулярные орбитали и векторы смещений ядер в процессе колебаний визуализировались с применением программ Molekel 4.3 [16] и GaussView [17].

\section{Результаты и их обсуждение}

\section{Структура $\mathrm{H}_{2}-\mathrm{Pz}(\mathrm{Ph})_{4}(\mathrm{CN})_{4}$ и их производных в основном и в возбужденных состояниях с учетом влияния среды в рамках метода РСМ}

Проведенные расчеты структуры $\mathrm{H}_{2}-\mathrm{Pz}(\mathrm{Ph})_{4}(\mathrm{CN})_{4}$ методом wB97XD/6-31g(d,p) предсказывают плоское строение макроцикла. Цианогруппы расположены в плоскости макроцикла, наклон фенильных колец по отношению к макроциклу составляет $\sim 30^{\circ}$ в толуоле и $\sim 34^{\circ}$ в воде. Замещение цианогрупп атомами $\mathrm{H}\left(\mathrm{H}_{2}-\mathrm{Pz}(\mathrm{Ph})_{4}\right)$ 
Таблица 1. Рассчитанные методом wB97XD/6-31g(d,p) с учетом растворителя (вода, РСМ) значения длин волн $(\lambda, \mathrm{nm})$ и сил осциллятора (в скобках) десяти переходов $\mathrm{H}_{2}-\mathrm{Pz}(\mathrm{p}-$ $\mathrm{F}-\mathrm{Ph})_{4}(\mathrm{CN})_{4}$ в воде, в конфигурационном составе указаны одноэлектронные возбуждения с наибольшим вкладом

\begin{tabular}{c|l}
\hline Переход & Вклады конфигураций, \% \\
\hline $644(0.55)$ & HOMO $\rightarrow$ LUMO $(87)$ \\
\hline $597(0.52)$ & HOMO $\rightarrow$ LUMO $+1(87)$ \\
\hline $421(0.11)$ & HOMO-2 $\rightarrow$ LUMO $+1(63)$ \\
\hline $420(0.00)$ & HOMO-1 $\rightarrow$ LUMO $+1(68)$ \\
\hline $385(0.33)$ & HOMO-2 $\rightarrow$ LUMO $(65)$ \\
\hline $385(0.00)$ & HOMO-3 $\rightarrow$ LUMO $(69)$ \\
\hline $363(0.00)$ & HOMO-1 $\rightarrow$ LUMO $(75)$ \\
\hline $356(0.39)$ & HOMO-4 $\rightarrow$ LUMO $+1(30)$ \\
\hline $342(1.17)$ & HOMO-4 $\rightarrow$ LUMO $(61)$ \\
\hline $329(0.00)$ & HOMO-3 $\rightarrow$ LUMO $+1(54)$
\end{tabular}

и фторирование фенильных колец в пара-положении $\left(\mathrm{H}_{2}-\mathrm{Pz}(\mathrm{p}-\mathrm{F}-\mathrm{Ph})_{4}(\mathrm{CN})_{4}\right)$ незначительно сказываются на молекулярной структуре. Расширение базисного набора до $6-3 \lg (2 \mathrm{~d}, 2 \mathrm{p})$ также к заметным изменениям в структуре не приводит.

Зависимость квантового выхода флуоресценции от вязкости у флуоресцентных роторов обычно связывается с заселением состояния с низким значением силы осциллятора перехода при значительном изменении положения частей молекулы по торсионной координате. Как правило, такие состояния характеризуются заметным перераспределением заряда между молекулярными фрагментами. В табл. 1 приведены рассчитанные электронные переходы (в воде) $\mathrm{H}_{2}-\mathrm{Pz}(\mathrm{p}-\mathrm{F}-\mathrm{Ph})_{4}(\mathrm{CN})_{4}$, для которого ранее была обнаружена зависимость квантового выхода флуоресценции от вязкости [6,7]. Расчетные данные удовлетворительно согласуются с максимумами полос поглошения $\mathrm{H}_{2}-\mathrm{Pz}(\mathrm{p}-\mathrm{F}-\mathrm{Ph})_{4}(\mathrm{CN})_{4}[18]$. С асимметричной полосой $612 \mathrm{~nm}$ с полушириной порядка $90 \mathrm{~nm}$ можно соотнести два рассчитанных вертикальных перехода 597 и $644 \mathrm{~nm}$, а с полосами 340 и $406 \mathrm{~nm}-$ переходы при 342 и $385 \mathrm{~nm}$.

Среди десяти нижних электронных переходов, приведенных в табл. 1, присутствуют три, для которых сила осциллятора перехода близка к нулю. Они относятся преимущественно к одноэлектронным возбуждениям с участием орбиталей НОМО-1 и НОМО-3, для которых большая часть электронной плотности локализована на фенильных кольцах пирролениновых и пиррольных колец соответственно (рис. 2). Вакантные орбитали, вовлеченные в эти переходы, преимущественно локализованы на макроцикле. По этой причине данные три перехода сопровождаются значительным перераспределением заряда. C учетом поляризующего влияния среды для соответствующих возбужденных состояний можно было ожидать значительного понижения энергии в результате релаксации. Однако проведенная оптимизация геометрии показала, что этот эффект недостаточно велик для того, чтобы данные возбужденные состояния могли принять участие в тушении флуоресцентного $S_{1}$-состояния. Связано это с тем, что орбитали НОМО-1 и НОМО-3 для связей СС между макроциклом и фенильными кольцами являются разрыхляющими. Как следствие, релаксация этих состояний сопровождается уменьшением угла наклона фенильных колец к макроциклу $\left(\sim 10^{\circ}\right)$, что уменьшает степень разделения заряда. Оптимизация геометрии в $S_{1}$-состоянии также приводит к небольшому уменьшению угла наклона фенильных колец по отношению к макроциклу $\left(3^{\circ}-6^{\circ}\right)$. При этом сила осциллятора перехода $S_{0} \rightarrow S_{1}$ практически не меняется.

Разделение заряда в молекуле $\mathrm{H}_{2}-\mathrm{Pz}(\mathrm{p}-\mathrm{F}-\mathrm{Ph})_{4}(\mathrm{CN})_{4}$ при фотофозбуждении возможно при условии взаимно перпендикулярного расположения одного или нескольких фенильных колец по отношению к макроциклу. В качестве такой модели нами была рассмотрена структура с расположением таким образом одного из фенильных кольц. Оптимизация геометрии в соответствующем состоянии с переносом заряда (СТ) проводилась при условии замораживания торсионного угла, определяющего ортогональное положение фенильного кольца. С целью предотвращения смешивания СТ-состояния с локально возбужденными состояниями и его стабилизации был выбран функционал B3LYP, который, как известно, существенно занижает положение СТ-переходов, и в качестве сольватной оболочки использовалась вода (РСМ-метод). Проведенный после процедуры оптимизации геометрии расчет колебаний показал, что данное состояние является неустойчивым.

Подобные результаты были получены для $\mathrm{H}_{2}$ $\mathrm{Pz}(\mathrm{Ph})_{4}(\mathrm{CN})_{4}$ и $\mathrm{H}_{2}-\mathrm{Pz}\left(\mathrm{o}-\mathrm{CH}_{3}-\mathrm{Ph}\right)_{4}(\mathrm{CN})_{4}$ (рис. 1). В целом они свидетельствуют о том, что зависимость флуоресцентных свойств соединения $\mathrm{H}_{2}-\mathrm{Pz}(\mathrm{Ph})_{4}(\mathrm{CN})_{4}$ и его производных от вязкости не связана с заселением в результате фотовозбуждения состояния с малым значением силы осциллятора перехода при условии заметного изменения положения фенильных колец по торсионной координате. По этой причине соединение $\mathrm{H}_{2}-\mathrm{Pz}(\mathrm{Ph})_{4}(\mathrm{CN})_{4}$ и его производные не могут быть отнесены к флуоресцентным роторам.

\section{Структура сольватных комплексов $\mathrm{H}_{2}-\mathrm{Pz}(\mathrm{Ph})_{4}(\mathrm{CN})_{4}$, его производных и порфиринового аналога}

Связи NH пиррольных колец и атомы N пирролениновых колец порфиразинов и порфиринов вовлечены во внутримолекулярные водородные связи [19]. С их участием возможно также образование сольватных комплексов с молекулами растворителей, что оказывает 

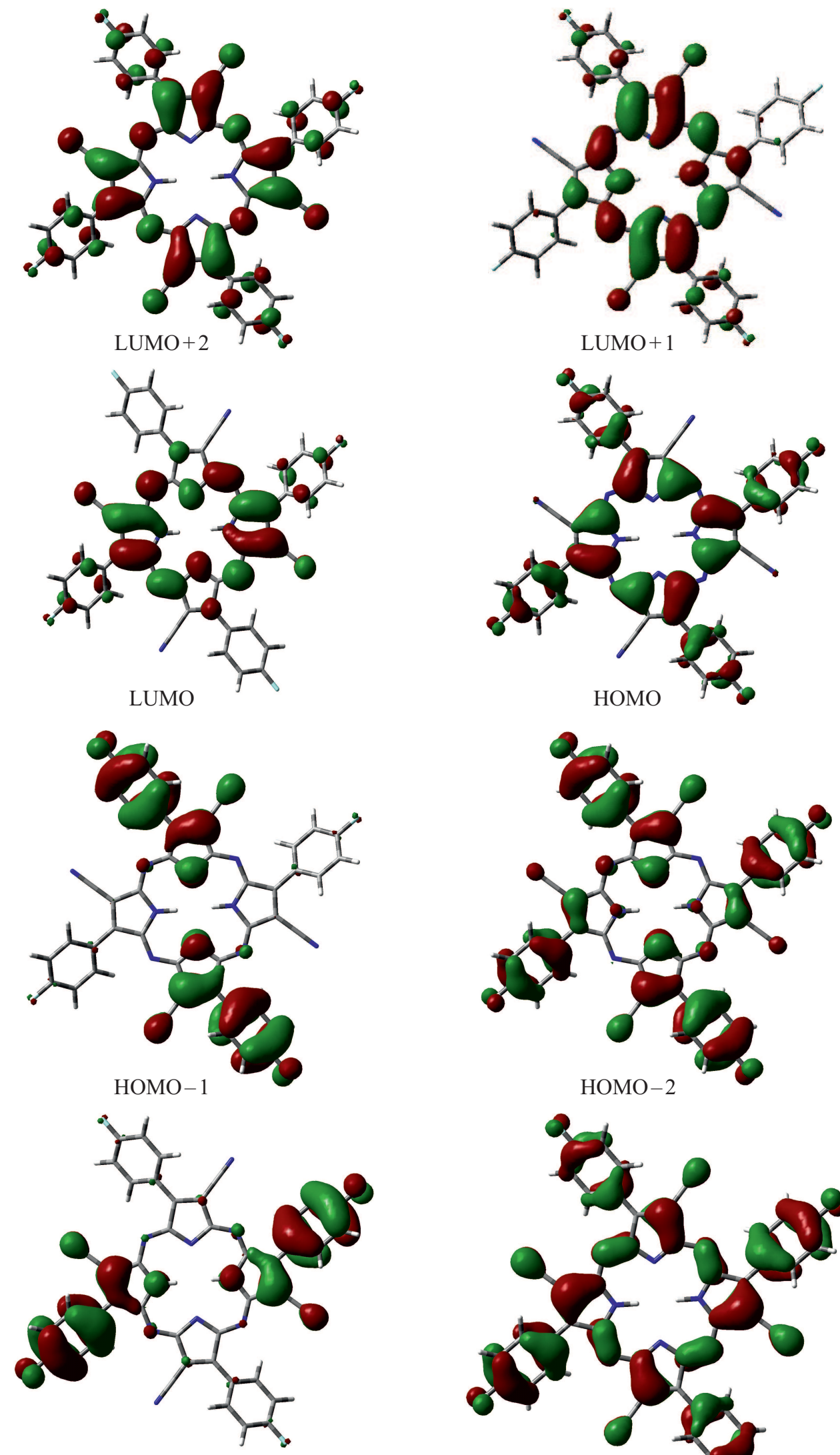

HOMO - 3

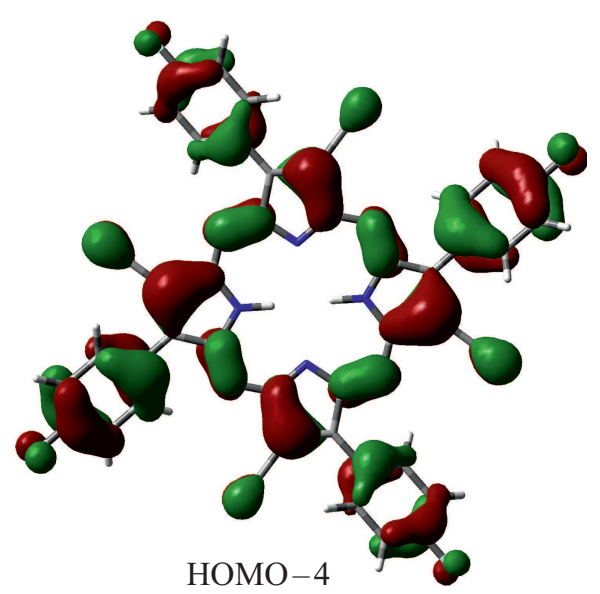

Рис. 2. Молекулярные орбитали, принимающие участие в формировании длинноволновых переходов $\mathrm{H}_{2}-\mathrm{Pz}(\mathrm{p}-\mathrm{F}-\mathrm{Ph})_{4}(\mathrm{CN})_{4}$ (табл. 1). 
Таблица 2. Структурные параметры водородных связей сольватных комплексов с водой $\mathrm{H}_{2}-\mathrm{Pz}(\mathrm{Ph})_{4}(\mathrm{CN})_{4}$, его производных и порфиринового аналога

\begin{tabular}{l|c|c|c}
\hline \multicolumn{1}{c|}{ Соединение } & $\begin{array}{c}d, \AA \\
\mathrm{H}_{2} \mathrm{O} \cdots \mathrm{NH}\end{array}$ & $\begin{array}{c}d, \AA \\
\mathrm{NH}\end{array}$ & $\begin{array}{c}d, \AA \\
\mathrm{H}_{2} \mathrm{O} \cdots \mathrm{N}\end{array}$ \\
\hline $\mathrm{H}_{2}-\mathrm{Pz}(\mathrm{Ph})_{4}(\mathrm{CN})_{4}$ & - & 1.011 & - \\
\hline $\mathrm{H}_{2}-\mathrm{Pz}(\mathrm{Ph})_{4}(\mathrm{CN})_{4} \oplus 2 \mathrm{H}_{2} \mathrm{O}$ & 2.844 & 1.022 & 3.047 \\
\hline $\mathrm{H}_{2}-\mathrm{Pz}(\mathrm{CN})_{4} \oplus 2 \mathrm{H}_{2} \mathrm{O}$ & 2.837 & 1.023 & 3.049 \\
\hline $\mathrm{H}_{2}-\mathrm{Pz}(\mathrm{Ph})_{4} \oplus 2 \mathrm{H}_{2} \mathrm{O}$ & 2.851 & 1.023 & 3.095 \\
\hline $\mathrm{H}_{2}-\mathrm{Pz} \oplus 2 \mathrm{H}_{2} \mathrm{O}$ & 3.151 & 1.009 & 3.156 \\
\hline $\mathrm{H}_{2}-\mathrm{P}(\mathrm{Ph})_{4}(\mathrm{CN})_{4} \oplus 2 \mathrm{H}_{2} \mathrm{O}$ & 2.836 & 1.025 & 2.959
\end{tabular}

влияние на структуру молекул в основном и в возбужденных состояниях [20]. Имея в виду это обстоятельство, использованная выше расчетная модель была расширена за счет включения в нее молекул растворителя в явном виде.

Проведенные расчеты показали, что $\mathrm{H}_{2}-\mathrm{Pz}(\mathrm{Ph})_{4}(\mathrm{CN})_{4}$ и его производные образуют сольватные комплексы с кислородсодержащими молекулами растворителя с участием пиррольных и пирролениновых колец. Такие комплексы, в частности, были обнаружены в воде, метаноле, этаноле, глицерине и тетрагидрофуране. При этом взаимодействие с молекулами растворителя сопровождается заметным искажением планарной структуры макроцикла. Ниже в качестве примера такого взаимодействия и влияния на него молекулярной структуры тетрапиррола будут рассмотрены сольватные комплексы $\mathrm{H}_{2}-\mathrm{Pz}(\mathrm{Ph})_{4}(\mathrm{CN})_{4}$ и его производных с водой, поскольку именно в этом растворителе было обнаружено наиболее сильное тушение флуоресценции [6].

Структура сольватного комплекса $\mathrm{H}_{2}-\mathrm{Pz}(\mathrm{Ph})_{4}(\mathrm{CN})_{4}$ с двумя молекулами воды $\left(\mathrm{H}_{2}-\mathrm{Pz}(\mathrm{Ph})_{4}(\mathrm{CN})_{4} \oplus 2 \mathrm{H}_{2} \mathrm{O}\right)$ по данным расчета с учетом растворителя (вода) приведена на рис. 3 (структуры $a, a^{\prime}$ ). Как можно видеть, порфиразиновый макроцикл искажен по типу седла [18]: пиррольные и пирролениновые кольца выходят из плоскости макроцикла в противоположных направлениях. Одна из молекул воды вовлечена в водородные связи $\mathrm{O}\left(\mathrm{H}_{2} \mathrm{O}\right) \cdots \mathrm{HN}$ с двумя пиррольными кольцами, вторая - в водородные связи $\mathrm{OH}\left(\mathrm{H}_{2} \mathrm{O}\right) \cdots \mathrm{N}$ с двумя пирролениновыми кольцами. В табл. 2 приведены расстояния между атомами $\mathrm{N}$ и $\mathrm{O}$, вовлеченными в данное взаимодействие. Их сравнение позволяет сделать вывод о том, что связь молекулы воды с пиррольными кольцами сильнее. Имея в виду данное обстоятельство, была предпринята попытка оптимизации структуры сольватного комплекса, у которого одна молекула воды взаимодействует с одним пиррольным кольцом, а вторая молекула воды - с другим на противоположной стороне макроцикла. Такая структура оказалась неустойчивой: в процессе оптимизации переходит в конформацию, рас- смотренную выше. Это связано (см. ниже) с заметным дипольным моментом седлообразной структуры $(\sim 8 \mathrm{D})$, который способствует понижению энергии сольватного комплекса за счет кулоновского взаимодействия с полярной средой.

С целью выяснения роли периферических заместителей во взаимодействии $\mathrm{H}_{2}-\mathrm{Pz}(\mathrm{Ph})_{4}(\mathrm{CN})_{4}$ с молекулами воды были рассчитаны структуры сольватных комплексов с другими производными порфиразина. Как оказалось, переход к порфиразину (фенильные и цианогруппы $\mathrm{H}_{2}-\mathrm{Pz}(\mathrm{Ph})_{4}(\mathrm{CN})_{4}$ заменены на атомы $\left.\mathrm{H}\right)$ приводит к существенной дестабилизации взаимодействия с молекулами воды, о чем свидетельствуют значения расстояния между атомами О молекул воды и атомами $\mathrm{N}$ пиррольных и пирролениновых колец, а также заметное уменьшение длины NH-связей (табл. 2). При этом седлообразная конформация становится неустойчивой и переходит в конформацию по типу волны [19] с симметричным расположением двух молекул воды относительно макроцикла. В случае замены на атомы Н цианогрупп седлообразная конформация макроцикла также претерпевает изменения. В данной структуре $\mathrm{H}_{2}-\left(\mathrm{Pz}(\mathrm{Ph})_{4}\right)$ реализуется водородная связь только с одним пиррольным кольцом. Соответственно неплоскостное искажение наблюдается только в этой части макроцикла (рис. 3, структуры $\left.b, b^{\prime}\right)$. Неплоскостное искажение по типу седла для $\mathrm{H}_{2}-\mathrm{Pz}(\mathrm{CN})_{4}$ по сравнению $\mathrm{c} \mathrm{H}_{2}-\mathrm{Pz}(\mathrm{Ph})_{4}(\mathrm{CN})_{4}$ усиливается, однако незначительно сказывается на положении молекул воды относительно центральных атомов азота макроцикла. Особенностью сольватных комплексов $\mathrm{H}_{2}-\mathrm{Pz}(\mathrm{Ph})_{4}(\mathrm{CN})_{4}, \mathrm{H}_{2}-\mathrm{Pz}(\mathrm{Ph})_{4}$ и $\mathrm{H}_{2}-\mathrm{Pz}(\mathrm{CN})_{4}$ с водой является существенное нарушение $s p^{2}$-гибридизации атомов азота пиррольных колец, которое сопровождается пирамидализацией его связей с атомами Н и С. В отсутствие сольватации двугранный угол $\mathrm{CCHN}$ c участием ближайших к атому $\mathrm{N}$ атомов равен нулю. Для сольватных комплексов $\mathrm{H}_{2}-\mathrm{Pz}(\mathrm{Ph})_{4}(\mathrm{CN})_{4} \oplus 2 \mathrm{H}_{2} \mathrm{O}$, $\mathrm{H}_{2}-\mathrm{Pz}(\mathrm{CN})_{4} \oplus 2 \mathrm{H}_{2} \mathrm{O}$ и $\mathrm{H}_{2}-\mathrm{Pz}(\mathrm{Ph})_{4} \oplus 2 \mathrm{H}_{2} \mathrm{O}$ он равен соответственно 9.9, 10.4 и $12.3^{\circ}$.

С целью выяснения причин указанных выше структурных отличий сольватных комплексов были проведены расчеты структуры и распределения электронной плотности для исходных соединений (без сольватирующих молекул). Как выяснилось, размеры внутреннего окна макроцикла у данных молекул отличаются не более, чем на $0.007 \AA$, а длины NH-связей совпадают (1.011 $\AA$ ), что указывает на одинаковую силу внутримолекулярных водородных связей. Соответственно по этой причине можно было ожидать и одинаковой структуры сольватных комплексов с молекулами воды. Поскольку это не так, имеющиеся отличия можно отнести на счет особенностей межмолекулярных взаимодействий с полярным окружением. Неплоскостное искажение данных молекул по типу седла способствует возникновению дипольного момента, направленного перпендикулярно макроциклу, и усиливает межмолекулярное взаимодействие со средой. Это позволяет обеспечить больший выход пирроль- 

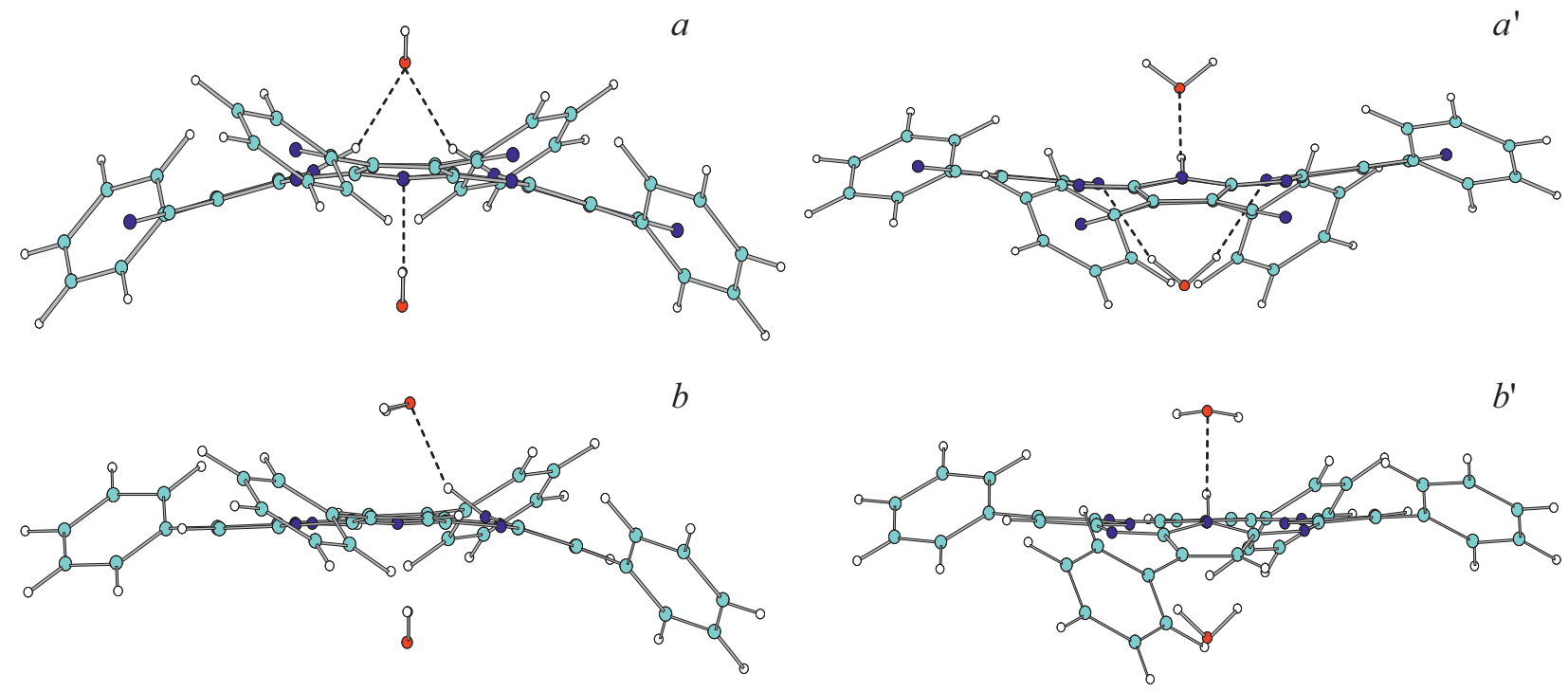

C
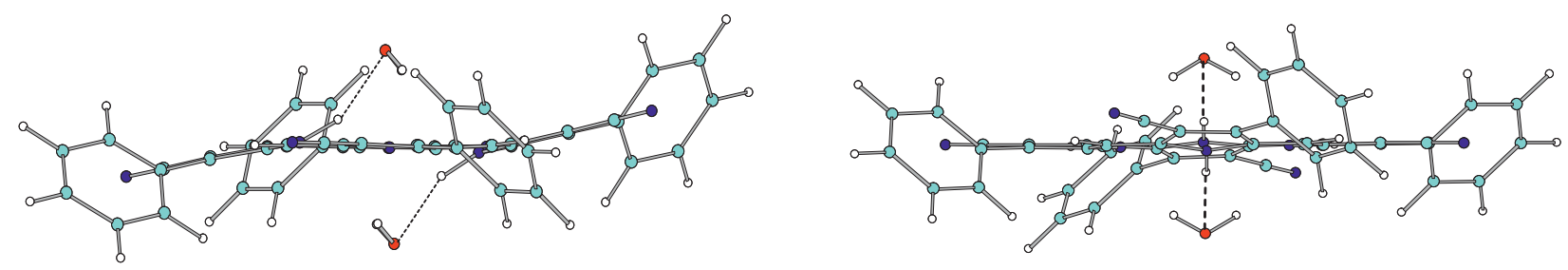

Рис. 3. Структура сольватных комплексов в основном состоянии по данным расчетов методом wB97XD/6-31g(d,p) c учетом растворителя (вода, $\mathrm{PCM}): a, a^{\prime}-\mathrm{H}_{2}-\mathrm{Pz}(\mathrm{Ph})_{4}(\mathrm{CN})_{4} \oplus 2 \mathrm{H}_{2} \mathrm{O} ; b, b^{\prime}-\mathrm{H}_{2}-\mathrm{Pz}(\mathrm{Ph})_{4} \oplus 2 \mathrm{H}_{2} \mathrm{O} ; c, c^{\prime}-\mathrm{H}_{2}-\mathrm{P}(\mathrm{Ph})_{4}(\mathrm{CN})_{4} \oplus 2 \mathrm{H}_{2} \mathrm{O}$.

ных колец из плоскости макроцикла, что благоприятствует взаимодействию с сольватирующей молекулой воды. Так, для $\mathrm{H}_{2}-\mathrm{Pz}(\mathrm{Ph})_{4}(\mathrm{CN})_{4}$ расчет сольватного комплекса с водой в газовой фазе дает значение расстояния между атомами $\mathrm{O}\left(\mathrm{H}_{2} \mathrm{O}\right)$ и $\mathrm{N}$ пиррольных колец, равное $2.97 \AA$, а для раствора в воде - $2.84 \AA$. В составе рассматриваемых соединений фенильные группы заряжены положительно, а цианогруппы - отрицательно. Соответственно отличаются варианты искажения макроцикла, способствующие кулоновскому взаимодействию с полярным окружением.

В структуре порфиразина порфириновые СН-группы метиновых мостиков заменены на атомы азота (рис. 1). С целью выяснения их роли в образовании сольватных комплексов $\mathrm{H}_{2}-\mathrm{Pz}(\mathrm{Ph})_{4}(\mathrm{CN})_{4}$ была проведена оптимизация геометрии комплекса с водой его порфиринового аналога $\mathrm{H}_{2}-\mathrm{P}(\mathrm{Ph})_{4}(\mathrm{CN})_{4} \oplus 2 \mathrm{H}_{2} \mathrm{O}$ (рис. 3, структуры $\left.c, c^{\prime}\right)$. В этом случае связывание с молекулами воды сохраняется, однако геометрия комплекса существенно изменяется. Седлообразная структура оказывается неустойчивой, и вместо нее образуется комплекс с двумя молекулами воды, расположенными симметрично по разные стороны макроцикла и вовлеченными в водородные связи с пиррольными кольцами. При этом неплоскостное искажение макроцикла соответствует деформации по типу волны [19]. Причина такой кон- формационной перестройки состоит в том, что замена атомов $\mathrm{N}$ в мезо-положении макроцикла на СН-группы сопровождается увеличением внутреннего окна макроцикла (на $\sim 0.24 \AA)$ и образование водородных связей молекулы воды сразу с двумя пиррольными кольцами становится энергетически невыгодным.

\section{Динамика сольватных комплексов тетраарилтетрацианопорфиразинов в $S_{1}$-состоянии и их флуоресцентные свойства}

Расчет вертикальных переходов сольватного комплекса $\mathrm{H}_{2}-\mathrm{Pz}(\mathrm{Ph})_{4}(\mathrm{CN})_{4} \oplus 2 \mathrm{H}_{2} \mathrm{O}$, как и в случае $\mathrm{H}_{2}-\mathrm{Pz}(\mathrm{Ph})_{4}(\mathrm{CN})_{4}$ и $\mathrm{H}_{2}-\mathrm{Pz}(\mathrm{p}-\mathrm{F}-\mathrm{Ph})_{4}(\mathrm{CN})_{4}$ (см. выше), не выявил низколежащих возбужденных состояний, заселение которых при уменьшении вязкости раствора может приводить к тушению флуоресценции. Вместе с тем обнаруженное неплоскостное искажение у молекулы $\mathrm{H}_{2}$ $\mathrm{Pz}(\mathrm{Ph})_{4}(\mathrm{CN})_{4} \oplus 2 \mathrm{H}_{2} \mathrm{O}$ в основном состоянии позволило предположить, что в возбужденном $S_{1}$-состоянии структура комплекса может претерпевать значительные конформационные изменения, оказывающие влияние на константы скорости безызлучательных переходов.

На рис. 4, а представлено наложение рассчитанных структур $\mathrm{H}_{2}-\mathrm{Pz}(\mathrm{Ph})_{4}(\mathrm{CN})_{4} \oplus 2 \mathrm{H}_{2} \mathrm{O}$ в воде (метод РCM) в $S_{1^{-}}$и основном состояниях. Как можно видеть, в 

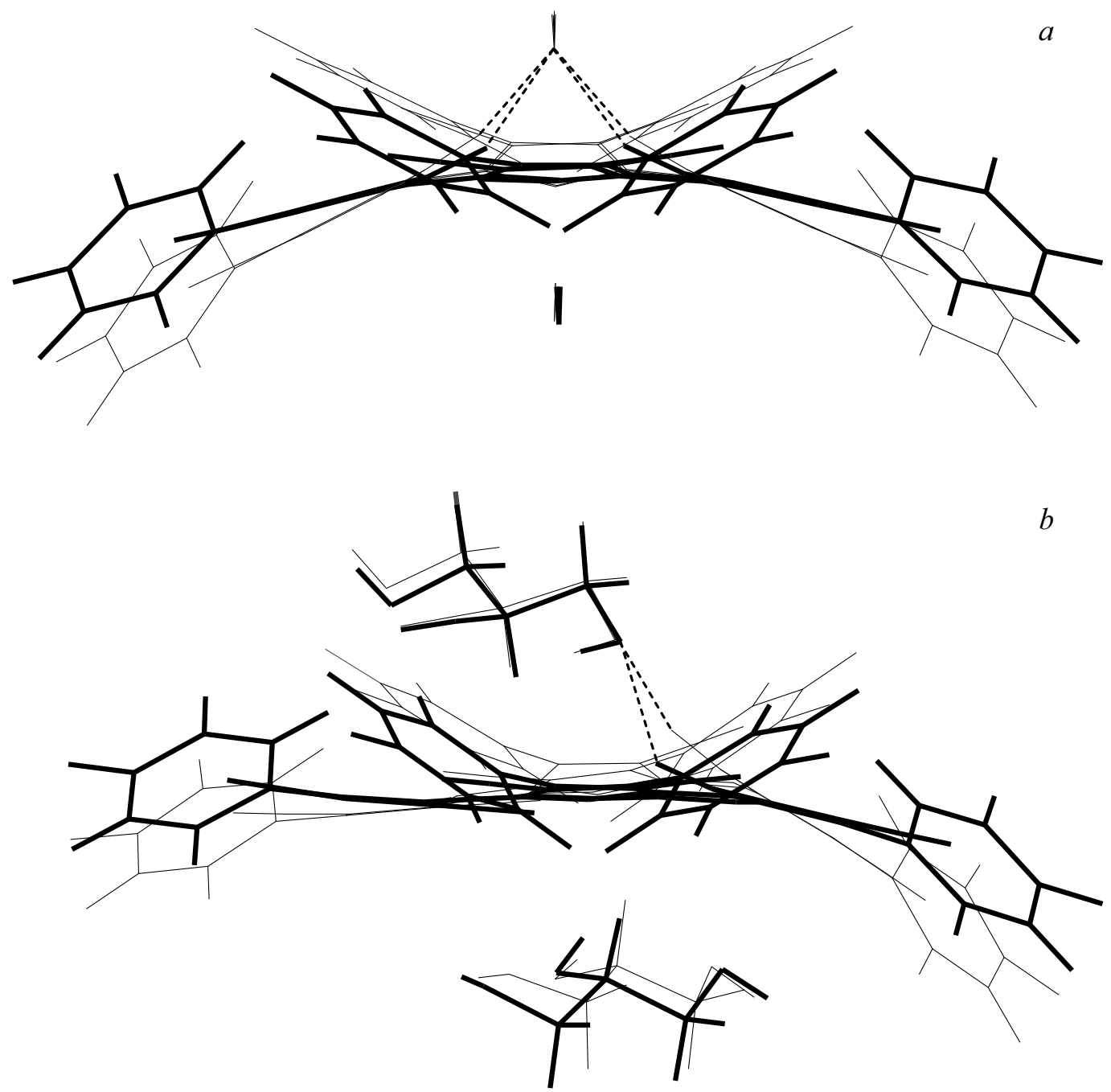

Рис. 4. Наложение структур в основном и $S_{1}$ (тонкие линии)-состояниях по данным оптимизации геометрии методами DFT и TDDFT (wB97XD/6-31g(d,p)): $a-\mathrm{H}_{2}-\mathrm{Pz}(\mathrm{Ph})_{4}(\mathrm{CN})_{4} \oplus 2 \mathrm{H}_{2} \mathrm{O} ; b-\mathrm{H}_{2}-\mathrm{Pz}(\mathrm{p}-\mathrm{F}-\mathrm{Ph})_{4}(\mathrm{CN})_{4} \oplus 2 \mathrm{C}_{3} \mathrm{H}_{8} \mathrm{O}_{3}$. Штриховыми линиями показаны водородные связи с участием NH-связей двух и одного пиррольного кольца соответственно.

$S_{1}$-состоянии седлообразное искажение молекулы существенно увеличивается. Оно сопровождается значительным смещением положения периферических заместителей. Для атомов азота цианогрупп оно составляет $0.8-0.85 \AA$, а для атомов Н фенильных колец в параположении - $1.33-1.43 \AA$ А. Существенное неплоскостное искажение комплекса $\mathrm{H}_{2}-\mathrm{Pz}(\mathrm{Ph})_{4}(\mathrm{CN})_{4} \oplus 2 \mathrm{H}_{2} \mathrm{O}$ в основном и в $S_{1}$-состояниях может способствовать тушению флуоресценции $\mathrm{H}_{2}-\mathrm{Pz}(\mathrm{Ph})_{4}(\mathrm{CN})_{4}$ в воде подобно тому, как это наблюдалось ранее для ряда стерически затрудненных порфиринов [21-29] и их сольватных комплексов [13]. Так, например, для порфиринов с искажением макроцикла по типу седла в толуоле при $273 \mathrm{~K}$ отмечалось уменьшение $\tau_{\mathrm{F}}$ почти в 20 раз [28]. Однако для $\mathrm{H}_{2}-$ $\mathrm{Pz}(\mathrm{Ph})_{4}(\mathrm{CN})_{4}$ квантовый выход флуоресценции варьирует существенно больше: от $\sim 0.003$ в воде до $\sim 0.5$ при связывании с водорастворимыми полимерами. Одна из причин этого может быть связана с тем, что взаимодействие с полимером способствует дезагрегации $\mathrm{H}_{2}$ -
$\mathrm{Pz}(\mathrm{Ph})_{4}(\mathrm{CN})_{4}$, который плохо растворяется в воде. Такой эффект наблюдался ранее, например при связывании хлорина е6 с поливинилпирролидоном [30]. Для $\mathrm{H}_{2-}$ $\mathrm{Pz}(\mathrm{Ph})_{4}(\mathrm{CN})_{4}$, насколько нам известно, литературные данные о спектроскопических и фотофизических проявлениях агрегации на данный момент отсутствуют.

Зависимость флуоресценции от вязкости для данной группы соединений была продемонстрирована при исследовании их метанол-глицериновых растворов $[6,7] \mathrm{c}$ различным соотношением компонент, а также растворов в глицерине при варьировании температуры [6]. При этом для $\mathrm{H}_{2}-\mathrm{Pz}(\mathrm{p}-\mathrm{F}-\mathrm{Ph})_{4}(\mathrm{CN})_{4}$ по сравнению с $\mathrm{H}_{2}-\mathrm{Pz}(\mathrm{o}-$ $\left.\mathrm{CH}_{3}-\mathrm{Ph}\right)_{4}(\mathrm{CN})_{4}$ (рис. 1) была обнаружена более выраженная зависимость $\tau_{\mathrm{F}}$ от вязкости раствора, что по мнению авторов подтверждает их отнесение к флуоресцентным роторам.

Агрегация тетрапирролов обычно достаточно хорошо проявляется в электронных спектрах поглощения, измерение которых позволяет ее обнаружить и контро- 
лировать. Если бы значительный рост времени жизни и квантового выхода флуоресценции $\mathrm{H}_{2}-\mathrm{Pz}(\mathrm{Ph})_{4}(\mathrm{CN})_{4}$ при увеличении температуры глицерина или его концентрации в метанол-глицериновой смеси был просто связан с дезагрегацией возможных агрегированных форм, то можно было бы ожидать заметного изменения спектра поглощения. Однако такие изменения обнаружены не были [6], что позволило авторам связать изменение флуоресцентных параметров с вязкостью.

По данным проведенных расчетов $\mathrm{H}_{2}-\mathrm{Pz}(\mathrm{Ph})_{4}(\mathrm{CN})_{4}$ образует сольватные комплексы и с метанолом, и с глицерином. При изменении концентрации компонент раствора может меняться не только вязкость, но и соотношение сольватных комплексов с молекулами метанола и глицерина. По этой причине флуоресцентные данные, полученные с использованием зависимости вязкости глицерина от температуры, могут быть более корректными.

На рис. 4, $b$ представлено наложение рассчитанных структур сольватных комплексов с глицерином $\mathrm{H}_{2}-\mathrm{Pz}(\mathrm{p}$ $\mathrm{F}-\mathrm{Ph})_{4}(\mathrm{CN})_{4} \oplus 2 \mathrm{C}_{3} \mathrm{H}_{8} \mathrm{O}_{3}$ в воде (метод РСМ) в основном и $S_{1}$-состояниях. Выбор воды в качестве среды связан с отсутствием в методе РСМ соответствующих параметров для глицерина и тем, что значения диэлектрической проницаемости для воды и глицерина близки. Как можно видеть, в данном случае для сольватного комплекса в основном состоянии реализуется структура с непланарным искажением макроцикла по типу волны. При этом в водородную связь с молекулой глицерина вовлечена $\mathrm{NH}$-связь лишь одного пиррольного кольца. Попытки получить сольватный комплекс с участием в водородной связи обоих пиррольных колец оказались безуспешными. При переходе в $S_{1}$-состояние деформация макроцикла заметно усиливается и изменяется положение всех периферических заместителей. Смещение их атомов неодинаково, и наибольшее значение имеет место для атома $\mathrm{F}$ того пиррольного кольца, которое вовлечено в водородную связь с молекулой глицерина (1.66 А).

$$
\text { Для сольватного }
$$

комплекса $\mathrm{H}_{2}-\mathrm{Pz}\left(\mathrm{o}-\mathrm{CH}_{3}-\mathrm{Ph}\right)_{4}(\mathrm{CN})_{4} \oplus 2 \mathrm{C}_{3} \mathrm{H}_{8} \mathrm{O}_{3} \quad$ также характерно искажение макроцикла по типу волны. Его величина несколько больше, чем у комплекса $\mathrm{H}_{2}-\mathrm{Pz}(\mathrm{p}-\mathrm{F}-\mathrm{Ph})_{4}(\mathrm{CN})_{4} \oplus 2 \mathrm{C}_{3} \mathrm{H}_{8} \mathrm{O}_{3}$, что может быть связано с расположением фенильных колец $\quad \mathrm{y} \quad \mathrm{H}_{2}-\mathrm{Pz}\left(\mathrm{o}-\mathrm{CH}_{3}-\mathrm{Ph}\right)_{4}(\mathrm{CN})_{4} \oplus 2 \mathrm{C}_{3} \mathrm{H}_{8} \mathrm{O}_{3}$ под большим углом к плоскости пиррольных и пирролениновых колец (на $\left.\sim 20^{\circ}\right)$. При переходе в $S_{1}$-состояние неплоскостное искажение комплекса $\quad \mathrm{H}_{2}-\mathrm{Pz}\left(\mathrm{o}-\mathrm{CH}_{3}-\mathrm{Ph}\right)_{4}(\mathrm{CN})_{4} \oplus 2 \mathrm{C}_{3} \mathrm{H}_{8} \mathrm{O}_{3}$ также увеличивается, но заметно меньше, чем для $\mathrm{H}_{2}-\mathrm{Pz}(\mathrm{p}-\mathrm{F}-\mathrm{Ph})_{4}(\mathrm{CN})_{4} \oplus 2 \mathrm{C}_{3} \mathrm{H}_{8} \mathrm{O}_{3}$. Если для последнего изменение двугранного угла, характеризующего наклон пиррольного кольца, вовлеченного в водородную связь с молекулой $\mathrm{C}_{3} \mathrm{H}_{8} \mathrm{O}_{3}$, составляет $\sim 20^{\circ}$, то для ортозамещенной производной $\sim 12^{\circ}$. Соответственно для
$\mathrm{H}_{2}-\mathrm{Pz}\left(\mathrm{o}-\mathrm{CH}_{3}-\mathrm{Ph}\right)_{4}(\mathrm{CN})_{4} \oplus 2 \mathrm{C}_{3} \mathrm{H}_{8} \mathrm{O}_{3} \quad$ при переходе $\quad$ в $S_{1}$-состояние имеют место меньшие смещения концевых атомов периферических заместителей.

При переходе рассматирваемых сольватных комплексов в равновесное $S_{1}$-состояние может реализовываться целый набор промежуточных конформаций с различным неплоскостным искажением. Время такого перехода очевидно зависит от вязкости. Чем она меньше, тем быстрее переход. Соответственно можно ожидать и меньшее среднее значение $\tau_{\mathrm{F}}$, поскольку неплоскостное искажение молекулярной структуры увеличивается. О влиянии таких деформаций на фотофизику тетрапирролов свидетельствуют как литературные данные [21-29,12,13] (см. выше), так и проведенный ниже анализ поведения констант скорости безызлучательной дезактивации $S_{1}$-состояния на примере сольватного комплекса $\mathrm{H}_{2}-\mathrm{Pz}(\mathrm{p}-\mathrm{F}-\mathrm{Ph})_{4}(\mathrm{CN})_{4} \oplus 2 \mathrm{C}_{3} \mathrm{H}_{8} \mathrm{O}_{3}$.

Изменение конформации сольватного комплекса при переходе из основного состояния в возбужденное и наоборот требует большего времени по сравнению со временем релаксации длин связей и валентных углов, поскольку предполагает значительные изменения в положении боковых заместителей и соответственно ближайших молекул растворителя. В этой связи можно предположить, что конформации в основном состоянии соответствует аналог в $S_{1}$-состоянии, а конформации в возбужденном состоянии - аналог в основном. С целью проверки этой возможности была рассчитана структура комплекса $\mathrm{H}_{2}-\mathrm{Pz}(\mathrm{p}-\mathrm{F}-\mathrm{Ph})_{4}(\mathrm{CN})_{4} \oplus 2 \mathrm{C}_{3} \mathrm{H}_{8} \mathrm{O}_{3}$ в основном состоянии при условии использования геометрии в $S_{1}$ состоянии в качестве исходной и замораживании ряда двугранных углов, определяющих положение периферических групп. Расчет колебаний для такой структуры показал отсутствие мнимых частот, что подтверждает данное предположение.

Рассчитанные значения энергетических зазоров $\Delta E\left(S_{0}-S_{1}\right)$ для конформации сольватного комплекса $\mathrm{H}_{2}-\mathrm{Pz}(\mathrm{p}-\mathrm{F}-\mathrm{Ph})_{4}(\mathrm{CN})_{4} \oplus 2 \mathrm{C}_{3} \mathrm{H}_{8} \mathrm{O}_{3} \quad$ в основном и в $S_{1}$-состояниях составили 13733 и $12683 \mathrm{~cm}^{-1}$ соответственно. Они определяют величину $\Delta E\left(S_{0}-S_{1}\right)$ сразу после фотовозбуждения (и быстрой релаксации длин связей и валентных углов) и после полной релаксации $S_{1}$-состояния, на скорость которой оказывает влияние вязкость растворителя. Для порфиринов и их аналогов ранее для зависимости константы скорости внутренней конверсии $k_{\text {ic }}$ от величины $\Delta E\left(S_{0}-S_{1}\right)$ использовалось выражение $k_{\text {ic }}=A \exp (\beta \Delta E)$ со значениями $\beta$, равными 0.0013 [12] и 0.0015 [13,20,31]. Предполагая такую же зависимость для порфиразинов, можно ожидать увеличения $k_{\text {ic }}$ не более, чем в 5 раз при переходе из начальной в равновесную конформацию $\mathrm{H}_{2}-\mathrm{Pz}(\mathrm{p}-\mathrm{F}-\mathrm{Ph})_{4}(\mathrm{CN})_{4} \oplus 2 \mathrm{C}_{3} \mathrm{H}_{8} \mathrm{O}_{3} \quad$ в $S_{1}$-состоянии. Такое усиление канала внутренней конверсии не может объяснить наблюдаемое уменьшение $\tau_{\mathrm{F}}$ в $\sim 13$ раз при изменении вязкости раствора $\mathrm{H}_{2}-\mathrm{Pz}(\mathrm{p}-\mathrm{F}-\mathrm{Ph})_{4}(\mathrm{CN})_{4}[6,7]$.

Приведенные выше рассуждения, однако, не учитывают, что для тетрапирролов большую роль в безызлуча- 
тельной дезактивации возбужденных состояний играют валентные NH-колебания [32,33]. Это связано с вовлеченностью NH-связей пиррольных колец во внутримолекулярные водородные связи, что усиливает ангармонизм $\mathrm{NH}$-колебаний. Для $\mathrm{H}_{2}-\mathrm{Pz}(\mathrm{p}-\mathrm{F}-\mathrm{Ph})_{4}(\mathrm{CN})_{4} \oplus 2 \mathrm{C}_{3} \mathrm{H}_{8} \mathrm{O}_{3}$ peлаксация $S_{1}$-состояния сопровождается значительным $(0.013 \AA)$ увеличением длины NH-связи одного из пиррольных колец за счет усиления межмолекулярной водородной связи с молекулой $\mathrm{C}_{3} \mathrm{H}_{8} \mathrm{O}_{3}$. В связи с этим можно ожидать большего, чем это следует из сравнения значений $\Delta E\left(S_{0}-S_{1}\right)$, усиления канала внутренней конверсии в результате конформационной перестройки $S_{1}$-состояния. Увеличение ангармонизма $\mathrm{NH}$-колебаний при переходе в $S_{1}$-конформацию приводит и к усилению канала безызлучательной дезактивации $S_{1}$ состояния за счет интерконверсии. Кроме того, можно ожидать увеличения константы скорости интерконверсии по причине большего неплоскостного искажения $\mathrm{H}_{2}-\mathrm{Pz}(\mathrm{p}-\mathrm{F}-\mathrm{Ph})_{4}(\mathrm{CN})_{4} \oplus 2 \mathrm{C}_{3} \mathrm{H}_{8} \mathrm{O}_{3}$ в $S_{1}$-конформации. Такое искажение приводит к появлению вклада $s p^{3}$ гибридизации на атомах углерода и азота макроцикла, что увеличивает спин-орбитальное взаимодействие между возбужденными состояниями.

Приведенные выше данные указывают на то, что зависимость $\tau_{\mathrm{F}} \quad$ от вязкости для $\mathrm{H}_{2}-\mathrm{Pz}(\mathrm{Ph})_{4}(\mathrm{CN})_{4}$ и их производных действительно может быть связана с конформационной динамикой их сольватных комплексов в $S_{1}$-состоянии. Величина данного эффекта зависит от того, насколько меняется структура макроцикла в $S_{1}$-состоянии при переходе из исходной конформации сольватного комплекса в равновесную. Поскольку для сольватного комплекса $\mathrm{H}_{2}-\mathrm{Pz}\left(\mathrm{o}-\mathrm{CH}_{3}-\mathrm{Ph}\right)_{4}(\mathrm{CN})_{4} \oplus 2 \mathrm{C}_{3} \mathrm{H}_{8} \mathrm{O}_{3}$ эти изменения меньше, чем для $\mathrm{H}_{2}-\mathrm{Pz}(\mathrm{p}-\mathrm{F}-\mathrm{Ph})_{4}(\mathrm{CN})_{4} \oplus 2 \mathrm{C}_{3} \mathrm{H}_{8} \mathrm{O}_{3}$ (см. выше), то для $\mathrm{H}_{2}-\mathrm{Pz}\left(\mathrm{o}-\mathrm{CH}_{3}-\mathrm{Ph}\right)_{4}(\mathrm{CN})_{4}$ следует ожидать меньшей чувствительности $\tau_{\mathrm{F}}$ к изменению вязкости раствора в соответствии с экспериментальными данными [6]. Учитывая отмеченные в предыдущем разделе особенности структуры, для комплексов $\mathrm{H}_{2}-\mathrm{Pz}(\mathrm{CN})_{4} \oplus 2 \mathrm{H}_{2} \mathrm{O}$ и $\mathrm{H}_{2}-\mathrm{Pz}(\mathrm{Ph})_{4} \oplus 2 \mathrm{H}_{2} \mathrm{O}$ также следует ожидать зависимость $\tau_{\mathrm{F}}$ от вязкости раствора. При этом она будет определяться не только изменением степени непланарности макроцикла в $S_{1}$-состоянии, но и размером периферических заместителей, поскольку от него зависит динамика молекулярной перестройки сольватной оболочки.

Изменение структуры сольватного комплекса $\mathrm{H}_{2}$ $\mathrm{P}(\mathrm{Ph})_{4}(\mathrm{CN})_{4} \oplus 2 \mathrm{H}_{2} \mathrm{O}$ в $S_{1}$-состоянии отличается от того, что имеет место для его порфиразинового аналога. В целом увеличение неплоскостного искажения в $S_{1}$-состоянии заметно меньше, при этом несколько больше для пирроленовых колец. Обращает на себя внимание также то, что длины NH-связей пиррольных колец не меняются. Связано это с различной природой $S_{1}$-состояния рассматриваемых соединений. У порфиразина, как и у $\mathrm{H}_{2}-\mathrm{Pz}(\mathrm{Ph})_{4}(\mathrm{CN})_{4} \oplus 2 \mathrm{H}_{2} \mathrm{O}$ в $S_{0} \rightarrow S_{1}$ переходе, доминирует вклад одноэлектронного возбуждения $\mathrm{HOMO} \rightarrow \mathrm{LUMO}$ (рис. 2). При этом нижняя вакантная орбиталь является разрыхляющей для $\mathrm{C}_{\alpha} \mathrm{N}$ связей на стороне пиррольных колец, что способствует усилению водородных связей с молекулой воды за счет выхода пиррольных колец из плоскости макроцикла. Для комплекса $\mathrm{H}_{2}-\mathrm{P}(\mathrm{Ph})_{4}(\mathrm{CN})_{4} \oplus 2 \mathrm{H}_{2} \mathrm{O}$ в конфигурационном составе $S_{0} \rightarrow S_{1}$-перехода доминирует вакантная орбиталь, заселение которой ослабляет $\mathrm{C}_{\alpha} \mathrm{C}_{m}$-связи на стороне пирроленинивых колец. Поскольку водородные связи молекулы воды с пирролениновыми кольцами слабее, чем с пиррольными, структурные изменения в $S_{1}$-состоянии для комплекса $\mathrm{H}_{2}-\mathrm{P}(\mathrm{Ph})_{4}(\mathrm{CN})_{4} \oplus 2 \mathrm{H}_{2} \mathrm{O}$ меньше. Можно предположить с учетом приведенных выше данных, что зависимость $\tau_{\mathrm{F}}$ от вязкости для $\mathrm{H}_{2}$ $\mathrm{P}(\mathrm{Ph})_{4}(\mathrm{CN})_{4}$ по сравнению с $\mathrm{H}_{2}-\mathrm{Pz}(\mathrm{Ph})_{4}(\mathrm{CN})_{4}$ будет заметно слабее.

Кинетики тушения флуоресценции $\mathrm{H}_{2}-\mathrm{Pz}(\mathrm{p}-\mathrm{F}-\mathrm{Ph})_{4}(\mathrm{CN})_{4} \quad$ и $\quad \mathrm{H}_{2}-\mathrm{Pz}\left(\mathrm{o}-\mathrm{CH}_{3}-\mathrm{Ph}\right)_{4}(\mathrm{CN})_{4} \quad$ как в метанол-глицериновом растворе, так и в глицерине не являются моноэкспоненциальными [6,7]. С учетом полученных в настоящей работе данных это можно связать с тем, что в растворе могут присутствовать сольватные комплексы с различными молекулами растворителя (метанол, глицерин, вода). Кроме того, в растворе могут находиться сольватные комплексы не с двумя, а с одной молекулой растворителя. Их структура может отличаться также вследствие различий во взаимном положении фенильных колец. Одной из возможных причин может быть наличие в $S_{1}$-состоянии большого набора конформаций, отличающихся степенью искажения макроцикла [12].

В заключение следует отметить следующее. В настоящей работе с использованием методов теории функционала плотности проведено исследование структуры $\mathrm{H}_{2}-\mathrm{Pz}(\mathrm{Ph})_{4}(\mathrm{CN})_{4}$ и его производных в основном и в ряде возбужденных состояний. Полученные данные указывают на то, что обнаруженная ранее для этих соединений с фотодинамической активностью зависимость времени жизни флуоресценции от вязкости не позволяет отнести их к флуоресцентным молекулярным роторам. Показано, что эта зависимость может быть связана со склонностью данных соединений образовывать сольватные комплексы с кислородсодержащими молекулами растворителя. Такое межмолекулярное взаимодействие по типу водородной связи приводит к нарушению плоскостности макроцикла, которое существенно усиливается в $S_{1}$-состоянии и сопровождается большим смещением периферических заместителей. Конформационная динамика в $S_{1}$-состоянии способствует уменьшению энергетического зазора $\Delta E\left(S_{0}-S_{1}\right)$, увеличению спинорбитального взаимодействия между возбужденными состояниями, а также ангармоничности валентных NHколебаний, которые играют большую роль в безызлучательной дезактивации тетрапирролов. Все эти факторы приводят к уменьшению времени жизни флуоресценции $\mathrm{H}_{2}-\mathrm{Pz}(\mathrm{Ph})_{4}(\mathrm{CN})_{4}$ и его производных в растворе и определяют зависимость времени жизни от вязкости 
среды. Выяснение количественного соотношения указанных выше вкладов в тушение флуоресценции требует дальнейших экспериментальных и теоретических исследований.

\section{Благодарности}

Автор благодарит Вычислительный центр НАН Беларуси за предоставленную возможность проведения расчетов.

\section{Финансирование работы}

Работа выполнена при частичной финансовой поддержке ГПНИ „ФЭИ $1.8^{\circ 6}$.

\section{Список литературы}

[1] Kuimova M.K., Botchway S.W., Parker A.W., Balaz M., Collins H.A., Anderson H.L., Suhling K., Ogilby P.R. // Nat. Chem. 2009. V. 1. N 1. P. 69-73.

[2] Vysniauskas A., Balaz M., Anderson H.L., Kuimova M.K. // Phys. Chem. Chem. Phys. 2015. V. 17. P. 7548-7554.

[3] Vysniauskas A., Qurashi M., Gallop N., Balaz M., Anderson H.L., Kuimova M.K. // Chem. Sci. 2015. V. 6. P. 5773-5778.

[4] Shimolina L.E., Izquierdo M.A., López-Duarte I., Bull J.A., Shirmanova M.V., Klapshina L.G., Zagaynova E.V., Kuimov M.K. // Sci. Rep. 2017. V. 7. P. 41097. doi 10.1038/srep41097

[5] Ширманова М.В., Балалаева И.В., Леканова Н.Ю., Мысягин С.А., Брилкина А.А., Клапшина Л.Г., Загайнова Е.В. // Биофизика. 2011. Т. 56. № 6. С. 1117-1124.

[6] Izquierdo M.A., Vyšniauskas A., Lermontova S.A., Grigoryev I.S., Shilyagina N.Y., Balalaeva I.V., Klapshina L.G., Kuimova M.K. // J. Mater. Chem. B. 2015. V. 3. N 6. P. $1089-1096$.

[7] Lermontova S.A., Grigoryev I.S., Shilyagina N.Yu., Peskova N.N., Balalaeva I.V., Shirmanova M.V., Klapshina L.G. // Russ. J. Gen. Chem. 2016. V. 86. N 6. P. 1330-1338.

[8] Lermontova S.A., Grigor'ev I.S., Ladilina E.Yu., Balalaeva I.V., Shilyagina N.Yu., Klapshina L.G. // Russ. J.Coord. Chem. 2018. V. 44. N 4. P. 301-315.

[9] Van Straten D., Mashayekhi V., de Bruijn H.S., Oliveira S., Robinson D.J. // Cancers. 2017. V. 9. N 2. P. 1-54.

[10] Frisch M.J., Trucks G.W., Schlegel H.B., Scuseria G.E., Robb M.A., Cheeseman J.R., Scalmani G., Barone V., Mennucci B., Petersson G.A., Nakatsuji H., Caricato M., Li X., Hratchian H.P., Izmaylov A.F., Bloino J., Zheng G., Sonnenberg J.L., Hada M., Ehara M., Toyota K., Fukuda R., Hasegawa J., Ishida M., Nakajima T., Honda Y., Kitao O., Nakai H., Vreven T., Montgomery Jr. J.A., Peralta J.E., Ogliaro F., Bearpark M., Heyd J.J., Brothers E., Kudin K.N., Staroverov V.N., Kobayashi R., Normand J., Raghavachari K., Rendell A., Burant J.C., Iyengar S.S., Tomasi J., Cossi M., Rega N., Millam N.J., Klene M., Knox J.E., Cross J.B., Bakken V., Adamo C., Jaramillo J., Gomperts R., Stratmann R.E., Yazyev O., Austin A.J., Cammi R., Pomelli C., Ochterski J.W., Martin R.L., Morokuma K., Zakrzewski V.G., Voth G.A., Salvador P.,
Dannenberg J.J., Dapprich S., Daniels A.D., Farkas Ö., Foresman J.B., Ortiz J.V., Cioslowski J., Fox D.J. Gaussian 09, Revision A.1, Gaussian Inc., Wallingford CT, 2009.

[11] Caricato M., Mennucci B., Tomasi J., Ingrosso F., Cammi R., Corni S., Scalmani G. // J. Chem. Phys. 2006. V. 124. P. 124520.

[12] Ивашин Н.В., Терехов С.Н. // Опт. и спектр. 2019. Т. 126. C. 285; Ivashin N.V., Terekhov S.N. // Opt. Spectrosc. 2019. V. 126. N 3. P. 205-215.

[13] Ивашин Н.В., Терехов С.Н. // Опт. и спектр. 2020. Т. 128. № 11. C. 1636-1645; Ivashin N., Terekhov S.N. // Opt. Spectrosc. 2020. V. 128. N 11. P. 1768-1777.

[14] Torres E., DiLabio G. // J. Phys. Chem. Lett. 2012. V. 3. P. 1738-1744.

[15] Caricato M., Mennucci B., Tomasi J., Ingrosso F., Cammi R., Corni S., Scalmani G. // J. Chem. Phys. 2006. V. 124. P. 124520.

[16] Flükiger P., Lüthi H.P., Portmann S., Weber J. Molekel 5.4.0.8. Swiss Center for Scientific Computing, Manno, Switzerland, 2009.

[17] Dennington R.D.II, Keith T., Millam J., Eppinnett K., Hovell W.L., Gilliland R. 2008 GaussView, Version 5.0 Semichem, Inc., Shawnee Mission, KS.

[18] Шилягина Н.Е. Автореф. дис. [Электронный ресурс] Режим доступа: http://www.science.vsu.ru/dissertations/672

[19] Ивашин Н.В., Пархоч О.П. // Опт. и спектр. 2004. Т. 97. № 3. C. 381-392; Ivashin N.V., Parkhots O.P. // Opt. Spectrosc. 2004. V. 97. N 3. P. 357-368.

[20] Ивашин Н.В., Щупак E.E. // Опт. и спектр. 2011. Т. 110. № 5. C. 741-751; Ivashin N.V., Shchupak E.E. // Opt. Spectrosc. 2011. V. 110. N 5. P. 694-704.

[21] Gentemann S., Medforth C.J., Forsyth T.P., Nurco D.J., Smith K.M., Fajer J., Holten D. // J. Am. Chem. Soc. 1994. V. 116. N 16. P. 7363-7368.

[22] Charlesworth P., Truscott T.G., Medforth C.J., Smith K.M. // Chem. Soc. Far. Trans. 1994. V. 90. N 8. P. 1073-1076.

[23] Gentemann S., Medforth C.J., Ema T., Nelson N.Y., Smith K.M., Fajer J., Holten D. // Chem. Phys. Lett. 1995. V. 245. P. 441-447.

[24] Shelhutt J.A., Song X.-Z., Ma J.-G., Jia S.-L., Jent-zen W., Medforth C.J. // Chem. Soc. Rev. 1998. V. 27. N 1. P. 31-42.

[25] Barkigia K.M., Nurco D.J., Renner M.W., Melamed D., Smith K.M., Fajer J. // J. Phys. Chem. B. 1998. V. 102. P. 322326.

[26] Chirvony V.S., van Hoek A., Galievsky V.A., Sazanovich L.V., Schaafsma T.J., Holten D. // J. Phys.Chem. B. 2000. V. 104. P. 9909-9917.

[27] Retsek J.L., Gentemann S., Medforth C.J., Smith K.M., Chirvony V.S., Fajer J., Holten D. // J. Phys. Chem. B. 2000. V. 104. P. 6690-6693.

[28] Lee J.-E., Yang J., Gunderson V.L., Wasielewski M.R., Kim D. // J. Phys. Chem. Lett. 2010. V. 1. N 1. P. 284-289.

[29] Сагун Е.И., Зенькевич Э.И., Кнюкшто В.Н., Панарин А.Ю., Семейкин А.С., Любимова Т.В. // Опт. и спектр. 2012. Т. 113. № 4. С. 431-443; Sagun E.I., Zenkevich E.I., Knyukshto V.N., Panarin A.Yu., Semeikin A.S., Lyubimova T.V. // Opt. Spectrosc. 2012. V. 113. N 4. P. $388-400$.

[30] Isakau H.A., Parkhats M.V., Knyukshto V.N., Dzhagarov B.M., Petrov E.P., Petrov P.T. // J. Photochem. Photobiol. B. 2008. V. 92. P. 165-174. 
[31] Chachisvilis M., Chirvony V.S., Shulga A.S., Kallenbring B., Larsson S., Sundstrom V. // J. Phys. Chem. 1996. V. 100. P. $13867-13873$.

[32] Burgner R.P., Ponte-Goncalver A.M. // J. Chem. Phys. 1974. V. 60. N 7. P. $2942-2944$.

[33] Соловьев К.Н., Кнюкшто В.Н., Цвирко М.П., Градюшко А.Г. // Опт. и спектр. 1976. Т. 41. № 6. С. 964-970. 